\title{
Applying Packets Meta data for Web Usage Mining
}

\author{
Prof. Dr. Alaa H. AL-Hamami \\ Amman Arab University for Graduate Studies, Zip Code: \\ 11953, P.O.B. 2234, Amman, Jordan, 2009. \\ Alaahamami@yahoo.com
}

\author{
Dr. Mohammad A. AL-Hamami \\ Delmon ,Bahrain, 2009
}

M_ah_1@yahoo.com,

\author{
Dr. Soukaena H. Hashem \\ University of technology, Iraq, 2009 \\ soukaena_hassan@yahoo.com,
}

\begin{abstract}
With data mining the techniques of the search engines and visitor tracking called web mining. The important task for web mining is web usage mining, which mines Web log records to discover user access patterns of Web pages. Analyzing and exploring regularities in Web log records identify potential customers for electronic commerce, enhance the quality and delivery of Internet information services to the end user, and improve Web server system performance. This research introduces a new approach for Mining Web Usage which aims to improve the mining results. For web usage mining we aim to mine the proposed added metadata of the packets in addition to the values of the header's fields of the packets which are used in web usage. This is done by adding metadata as a new factor in web logging process. These data about data (metadata) will be added as a new attributes in web databases. Even so that will increase the number of attributes; we will treat this problem by using a suitable method for finding frequent itemsets with limited time.
\end{abstract}

Keywords: Data mining, Web usage, Sensitive data mining, Meta data, and Internet packet.

\section{INTRODUCTION}

A web server usually registers a (Web) log entry, or Web log entry, for every access of a Web page. It includes the requested URL, the IP address from which the request is originated, and a timestamp. For Web-based e-commerce servers, a huge number of Web access log records are being collected. Popular Web sites may register the Web log records in order of hundreds of megabytes every day. Since Web log data provide information about what kind of users will access what kind of Web pages, Web log information can be integrated with Web content and Web linkage structure mining. This information will help Web page ranking, Web document classification, and the construction of a multilayered Web information base as well. With the enormous amount of data stored in files, databases, and other repositories, it is increasingly important, if not necessary, to develop powerful means for analysis and perhaps 
interpretation of such data and for the extraction of interesting knowledge that could help in decision-making [3].

Data Mining, also popularly known as Knowledge Discovery in Databases (KDD), refers to the nontrivial extraction of implicit, previously unknown and potentially useful information from data in databases. While data mining and knowledge discovery in databases (or KDD) are frequently treated as synonyms, data mining is actually part of the knowledge discovery process. Data mining techniques can be used to detect unusual patterns for user behavior that use the internet. Data mining should be applicable to any kind of information repository. World Wide Web is the most heterogeneous and dynamic repository available. World Wide Web is comprised of three major components: the content of the Web, which encompasses documents available; the structure of the Web, which covers the hyperlinks and the relationships between documents; and the usage of the web, describing how and when the resources are accessed [1, 2, 3].

\section{ASSOCIATION RULES ALGORITHM}

The efficient data mining techniques for web usage is association rules algorithms, the famous one of them is apriori algorithm. The problem is stated as follows, see table 1 , Let $I=\{i 1$, i2, ...im\} be a set of literals, called items. Let $D$ be a set of transactions, where each transaction $T$ is a set of items such that $T \subseteq I$. A unique identifier TID is given to each transaction. A transaction $T$ is said to contain $X$, a set of items in $I$, if $X \subseteq T$. An association rule is an implication of the form " $X \Rightarrow Y$ ", where $X \subseteq I, Y \subseteq I$, and $X \cap Y=\varnothing$. The rule $X \Rightarrow Y$ has a support $s$ in the transaction set $D$ is $s \%$ of the transactions in $D$ contain $X \cup Y$. In other words, the support of the rule is the probability that $X$ and $Y$ hold together among all the possible presented cases. It is said that the rule $X \Rightarrow Y$ holds in the transaction set $D$ with confidence $c$ if $c \%$ of transactions in $D$ that contain $X$ also contain $Y$. The problem of discovering all association rules from a set of transactions $D$ consists of generating the rules that have a support and confidence greater that given thresholds [4].

\section{MAXIMAL FREQUENT Itemset ALGORITHM (MAFIA)}

MAFIA is an algorithm for mining maximal frequent itemsets from a transactional database. Algorithm is especially efficient when the itemsets in the database are very long. The search strategy of algorithm integrates a depth-first traversal of the itemset lattice with effective pruning mechanisms. Implementation of the search strategy combines a vertical bitmap representation of the database with an efficient relative bitmap compression schema. In a thorough experimental analysis of algorithm on real data, isolate the effect of the individual components of the algorithm. Performance numbers show that algorithm outperforms previous work by up to an order of magnitude [5]. 


\subsection{Candidate Itemset Tree}

The process of generating candidate itemsets is done using a depth-first search, and the process can be represented as a candidate itemset tree. With each step down the tree, a single item is extended onto an itemset. As the itemsets grow larger and larger, the percentage of customers who have the itemset, or the support \%, will grow smaller and smaller. Eventually, this support value will go below the minimum support required for an itemset to be deemed frequent. When looking at the lexicographic tree, it is possible to draw a line that crosses all points at which an occurrence of an itemset being extended goes from frequent to infrequent. All itemsets directly above this line are termed the maximal frequent itemsets. By the Apriori principle, no itemset extensions below this line can be frequent since they all contain other itemsets within them that were found to be infrequent.

\subsection{Search Space Pruning}

In certain cases, branches of the candidate itemset tree can be "pruned" away, leading to fewer itemsets that need to be checked, and therefore a faster running time. This section explains what each of these pruning steps do.

Parent Equivalence Pruning - If an itemset in the tree has the same support as one of its candidate extensions then it can be pruned from the tree because it must only occur in the database as part of that candidate extension.

HUTMFI Superset Pruning - If the union of an itemset and its leftmost tail on the ordered subtree is frequent then the entire subtree can be pruned away. This process checks the current list of maximal frequent itemsets to see if this head-union-tail is already on this list.

FHUT - Frequent Head-Union-Tail - This pruning method is identical to HUTMFI except it actually checks the support of the HUT rather than searching to see if it is already in the MFI list. FHUT has been found to yield fewer performance increases than HUTMFI.

\subsection{Vertical Bitmap Representation}

MAFIA efficiently stores the transactional database as a series of vertical bitmaps, where each bitmap represents an itemset in the database and a bit in each bitmap represents whether or not a given customer has the corresponding itemset. Initially, each bitmap corresponds to a 1itemset, or a single item. The itemsets that are checked for frequency in the database become recursively longer and longer, and the vertical bitmap representation works perfectly in conjunction with this itemset extension. For example, the bitmap for the itemset $(a, b)$ can be constructed simply by performing an AND operation on all of the bits in the bitmaps for (a) and (b). Then, to count the number of customers that have (a,b), all that needs to be done is count the number of one bits in the $(a, b)$ bitmap equals the number of customers who have $(a, b)$. 
Clearly, the bitmap structure is ideal for both candidate itemset generation and support counting [5].

\section{THE PROPOSED SYSTEM}

In this research we describe a data mining framework for constructing web usage model. The key ideas are to mine web log record data for consistent and useful patterns of program and user behavior. So we could apply the web usage with high precision. We propose to use the association rules and frequent itemsets computed from logging data as the basis for guiding web usage. The general algorithm and all the details would be explained in the following sections briefly:

Traditionally web usage logging depends only on the values of packet's fields, such as local IP, local port, remote IP, remote port, state, type of protocol and time stamp. The traditional web database frame would be as in figure (1). For preparing the attributes for mining we giving each one a letter to present it is appearance such that if type of protocol is TCP then F will appear else F doesn't appear.

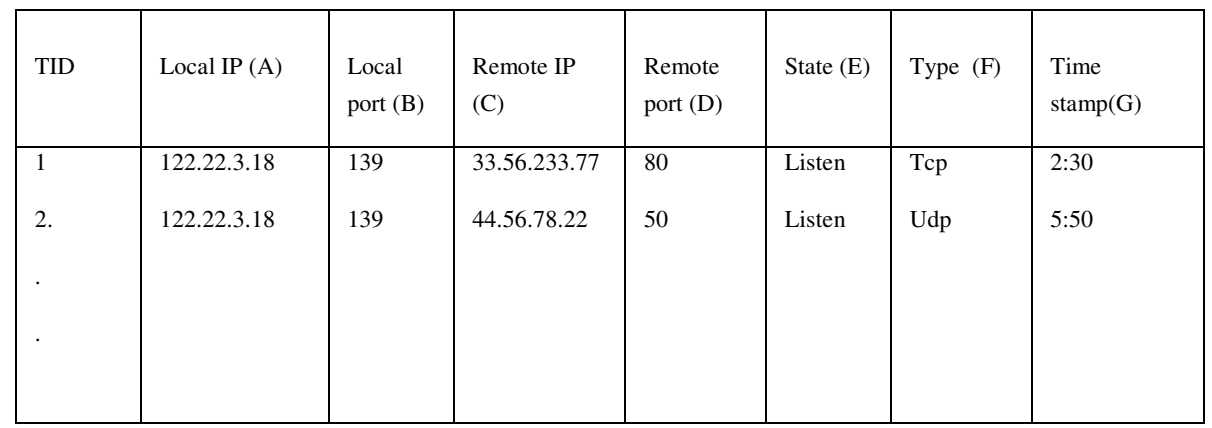

FIGURE 1 : Web logging database

Now we propose to add the metadata of each packet as a factor in web logging process. Then add this metadata (data about data) into the original web logging database as a new attributes. The new proposed database (which consists of traditional attributes and the new metadata attributes) will be mined. This will produce new important results. After adding the metadata as an attributes for the web logging database, the new frame of it will be as in figure 2 .

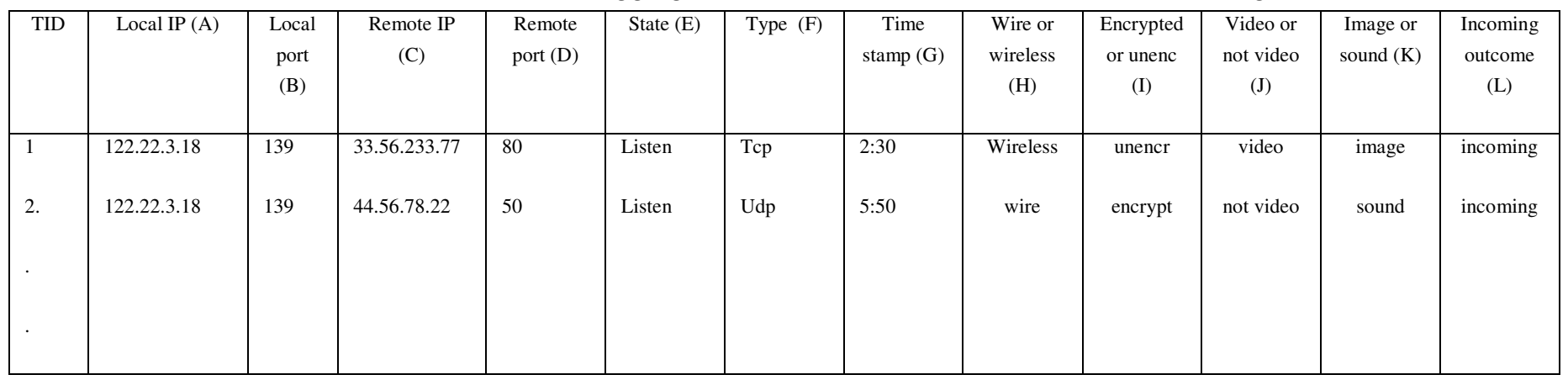

FIGURE 2: Web logging database with Metadata 


\section{IMPLEMENTATION}

To implement the suggestion, we must discuss an important aspect. This aspect discusses the case of adding metadata to the original database as a new attributes. This will make the number of attributes increase then that will increase the time of computations to find the frequent itemsets. This may be considered as a weak point in the proposal. The weakness can be broken by using MAFI method for finding the frequent itemsets instead of the traditional method which is used in apriori association rule mining. Now the implementation will be as in figure 3 .

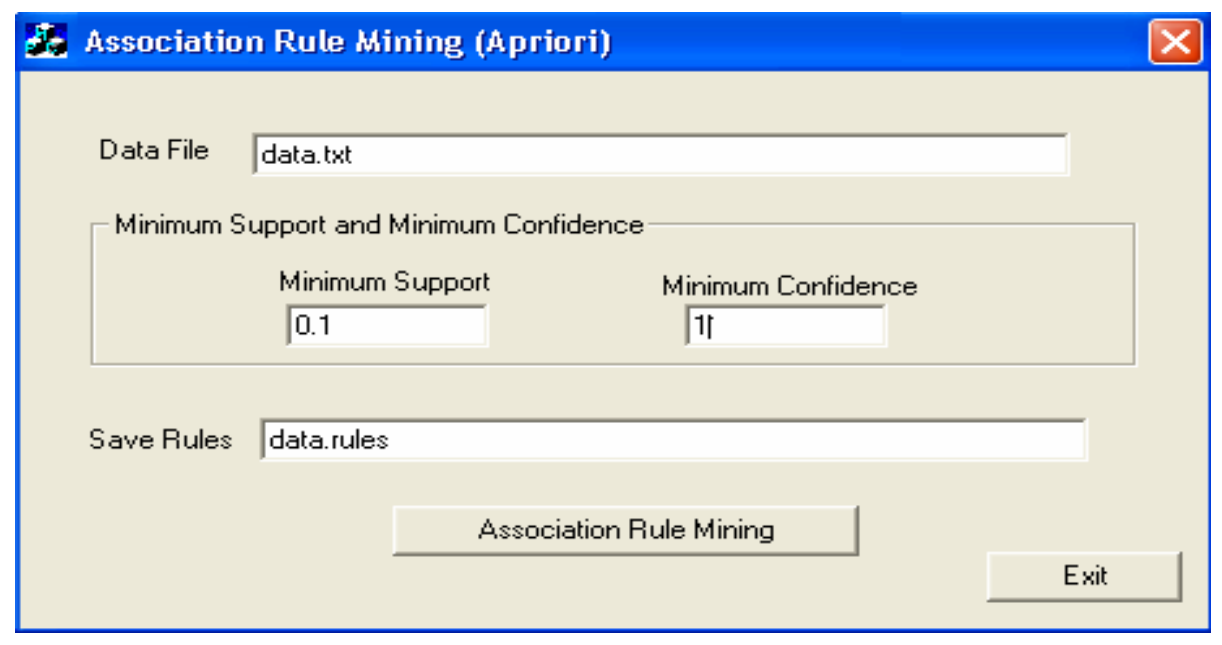

FIGURE 3: Association rule mining using MAFI

When the apriori software has been implemented on traditional web log database a collection of association rules has been resulted. As example of these rules:

\section{$\mathrm{AB} \rightarrow \mathrm{EF}$}

The resulted rule will be passed to the analysis stage. The result of association rules analysis that got from mining the traditional web log databases as follows:

For the 24 hours covered on 13-des-2008:

- 5 a.m. -6 a.m. = 23 visitors enter by UDP protocol on port 80 from EUR countries only for games.

- 6 a.m. -7 a.m. $=35$ visitors from different countries for email.

- 7 a.m. -8 a.m. = 42 visitors from Arabian for newsgroup.

- 8 a.m. -9 a.m. $=69$ visitors almost those are merchants dealing only with e-commerce.

- 9 a.m. -10 a.m. $=105$ visitors those using searching techniques for some of their interested subjects.

- 10 a.m. -11 a.m. = 323 visitors shopping.

When the apriori software implemented as in figure 3 on the proposed web log database a collection of association rules will be resulted. As example of these rules:

\section{$\mathrm{ABEF} \rightarrow \mathrm{IX}$}


The resulted rule will be passed to analysis stage for association rules that got from mining the proposed web log databases as follows:

For the 24 hours covered on 13-des-2008:

- 5 a.m. -6 a.m. = 23 visitors enter by UDP protocol on port 80 from EUR countries only for games. These packets are incoming, unencrypted, also they are video packets.

- 6 a.m. -7 a.m. = 35 visitors from different countries for email. These packet are incoming, encrypted, also they are text packets.

- 7 a.m. -8 a.m. = 42 visitors from Arabian for newsgroup. These packets are incoming, unencrypted; they are video, image and text packets.

- 8 a.m. -9 a.m. $=69$ visitors almost those are merchants dealing only with e-commerce. These packets are incoming, encrypted, they are text packets.

- 9 a.m. -10 a.m. = 105 visitors those using searching techniques for some of their interested subjects. These packets are outgoing, unencrypted; they are video, text, image packets.

- 10 a.m. - 11 a.m. = 323 visitors shopping. These packets are outgoing, unencrypted, they are images packets.

\section{CONCLUSION}

From the proposed research we conclude the following:

1. Implementation of the web usage is a great help for support the reputation of the site through tracking the visitors and reconfigure the server organization according to it.

2. From these discovered patterns by DM algorithm the web site administration would be able to depend on these patterns for reconfigure their site servers according to the way preferred by visitors, and almost make their predictions about the track of visitors in the coming times.

3. The suggestion which implies adding a metadata as a new attributes to the original databases attributes give much power pattern support accurate predictions.

4. By using MAFI technique in finding the frequent itemsets that break the weak of increase the computation of finding the association rules. So the proposal will save time and space.

\section{REFERENCES}

[1]. Hand D., Manila H. and Smyth p., "Principle of DM", the MIT Press publisher, 2001.

[2]. Mitra S., and Ahharya t., "Data Mining Multimedia, Soft Computing, and Bioinformatics", John Wiley and sons, Inc., 2003.

[3]. Arotaritei D. and Mitra S., "Web Mining: a Survey in the Fuzzy Framework", Edited by Human Factors and Ergonomics, 2003. 
[4]. Kantardzic M., "DM Concepts, Models, Methods and Algorithms", jhon wiley \&Sons, 2003.

[5]. Doug Burdick, Manuel Calimlim, Johannes Gehrke, “MAFIA: A Maximal Frequent Itemset Algorithm forTransactional Databases" Department of Computer Science, Cornell University, 2003.

[6]. Ala'a H. AL-Hamami, Mohammad Ala'a Al-Hamami and Soukaena Hassan Hasheem, “ Applying data mining techniques in intrusion detection system on web and analysis of web usage", Asian Journal of Information Technology, 2006.

[7]. Ala'a H. AL-Hamami, and Soukaena Hassan Hasheem," Privacy Preserving for Data Mining Applications", journal of technology, baghdad, Iraq, university of technology, 2008.

[8]. Mohammad A. Al- Hamami and Soukaena Hassan Hashem, " Applying Data Mining Techniques to Discover Methods that Used for Hiding Messages Inside Images ", The IEEE First International Conference on Digital Information Management (ICDIM2006), Bangalore, India, 2006. 